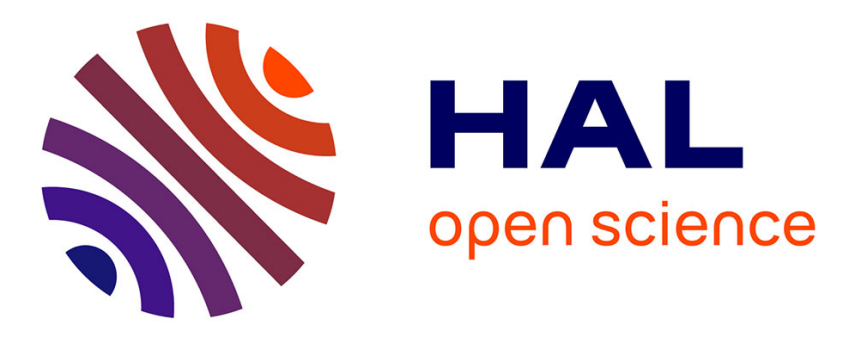

\title{
Transmit Antenna Selection for Coded Multiple-Input Dual-Output Systems
}

\author{
Ammar El Falou, Charlotte Langlais, Walaa Hamouda, Charbel Abdel Nour, \\ Catherine Douillard
}

\section{- To cite this version:}

Ammar El Falou, Charlotte Langlais, Walaa Hamouda, Charbel Abdel Nour, Catherine Douillard. Transmit Antenna Selection for Coded Multiple-Input Dual-Output Systems. ISTC 2014: 8th International Symposium on Turbo Codes and Iterative Information Processing, Aug 2014, Bremen, Germany. pp.269 - 273, 10.1109/ISTC.2014.6955127 . hal-01170234

\section{HAL Id: hal-01170234 \\ https://hal.science/hal-01170234}

Submitted on 17 Feb 2020

HAL is a multi-disciplinary open access archive for the deposit and dissemination of scientific research documents, whether they are published or not. The documents may come from teaching and research institutions in France or abroad, or from public or private research centers.
L'archive ouverte pluridisciplinaire HAL, est destinée au dépôt et à la diffusion de documents scientifiques de niveau recherche, publiés ou non, émanant des établissements d'enseignement et de recherche français ou étrangers, des laboratoires publics ou privés. 


\title{
Transmit Antenna Selection for Coded Multiple-Input Dual-Output Transmissions
}

\author{
Ammar El Falou ${ }^{\dagger}$, Charlotte Langlais ${ }^{\dagger}$, Walaa Hamouda ${ }^{\ddagger}$, Charbel Abdel Nour ${ }^{\dagger}$ and Catherine Douillard ${ }^{\dagger}$ \\ ${ }^{\dagger}$ Institut Mines-Telecom; Telecom Bretagne; UMR CNRS 3192 Lab-STICC \\ Electronics Department, Technopôle Brest Iroise CS 83818, 29238 Brest Cedex 3 \\ $\ddagger$ Concordia University, Department of Electrical and Computer Engineering \\ Montreal, Quebec, H3G 1M8, Canada \\ Email: \{ammar.elfalou, charlotte.langlais, charbel.abdelnour, catherine.douillard\}@telecom-bretagne.eu, \\ hamouda@ece.concordia.ca
}

\begin{abstract}
Antenna selection (AS) has been introduced to benefit from MIMO gains with tolerable cost and complexity where the transmission is only done via best antennas. In the literature, the AS has been addressed only for the case of uncoded MIMO systems. In order to be closer to practical systems, we assess in this paper the benefits of transmit AS technology for coded multiple-input dual-output (MIDO) systems. We start by designing space time codes (STCs) suitable for coded MIDO systems employing the transmit AS. Furthermore, we show that transmit AS provides a substantial SNR gain of more than $3 \mathrm{~dB}$ with respect to a conventional $2 \times 2$ turbo coded WiMAX system, and this, with a low cost and complexity increase.
\end{abstract}

\section{INTRODUCTION}

Most of modern wireless communication systems as WiMAX, WiFi, LTE and LTE-A have adopted the use of multiple antennas at the transmitter and the receiver, called multiple-input multiple-output (MIMO). Indeed, MIMO coding potential benefits are data rate increase and better reliability. Spatial multiplexing techniques [1] have been incorporated for data rate increase whereas space-time block codes (STBCs) $[2,3]$ have been designed to improve the channel reliability through spatial diversity. Multiplexing and diversity gains can be simultaneously delivered with a fundamental tradeoff between them [4-6].

Full-rate full-diversity STBCs for MIMO systems are conventionally designed according to the well-known asymptotic rank-determinant criteria [2]. These criteria aim to maximize the diversity gain and the coding gain of designed STBCs at high signal to noise ratio (SNR). However, STBCs designed to perform well at high SNRs do not guarantee a good behavior at low SNRs. Powerful forward error correction (FEC) codes, usually present in the transmission chain, achieve iterative convergence at low to moderate SNRs. FEC decoding is extremely sensitive to its input bitwise mutual information (BMI) value. The higher the BMI at the input of the FEC decoder, the lower the error rate at the output of the decoder. Therefore, we have proposed in [7,8] a non-asymptotic STC design criterion based on the BMI maximization at a specific target SNR.

In $[7,8]$, we have examined the improvement of standardized STBCs for coded MIMO systems with dual antennas at transmitter and receiver sides. Telecommunication standards define the use of more than dual antennas, e.g., up to $4 \times 4$ for the WiMAX and $8 \times 8$ for the LTE-A [9]. In contrast, the use of MIMO techniques lead to a complexity and cost increase with respect to SISO systems at both transmitter and receiver sides. Transmitting on multiple antennas require multiple analog radio-frequency (RF) chains which comprise expensive hardware blocks. The implementation of a high number of RF chains is not always possible due to physical and economic constraints, e.g., small handsets. Furthermore, the detection complexity increases with the number of transmit antennas for a full-rate transmission [8].

Antenna selection (AS) techniques have been proposed as a means to benefit from expected MIMO gains with tolerable cost and complexity [10-13]. Here, transmission and reception are performed only through a subset of the available antennas, thereby reducing the system complexity and the number of required RF chains. In our work, we focus on AS at the transmitter side. Indeed, AS at the receiver side is not recommended as it can lead to a considerable reduction in the system capacity. Transmit AS is a good approach to benefit from MIMO system gains with low cost and complexity [14]. For a MIMO system employing the AS technology, it has been shown that FD STBCs like the Alamouti code [3] can benefit from the maximal channel diversity, i.e., $N_{t} N_{r}$ at high SNRs [12]. In this paper, we focus on the study transmit AS for practical coded MIMO communication systems precisely systems having dual receive antennas or multiple input dual output (MIDO) systems. In order to compare this work with works in $[7,8]$, we consider as a reference for gains assessment a $2 \times 2$ turbo coded WiMAX system.

The remainder of this paper is organized as follows. We start in Section II by presenting the MIMO system model under consideration, the transmit antenna selection technology and the employed selection algorithm. In Section IV, we study the transmit AS for uncoded MIDO systems. In Section V, we design efficient adaptive STBCs for coded MIMO system with AS. Afterwards, we assess the gain provided by an AS on transmit antennas, with respect to classical WiMAX coded system. Section VI concludes the paper. 


\section{System MOdEL AND NOTATIONS}

We consider a coded MIMO system with $N_{t}$ transmit antennas, $N_{r}$ receive antennas operating over a quasi-static uncorrelated flat Rayleigh fading channel, i.e., the channel changes for each transmitted MIMO codeword of length $T$. A perfect channel state information (CSI) is assumed at the receiver, but not at the transmitter. We assume that a lowrate error-free zero-delay feedback channel feeds the set of best transmit antennas to the transmitter every $T$ channel use periods. This set is determined by a selection algorithm operating at the receiver side. The system is described as follows (see Fig. 1):

Transmitter side: an information word $\mathrm{b}$ is encoded via $\mathrm{a}$ powerful FEC code $\mathrm{C}$ with rate $R_{c}$. Resulting codeword is interleaved with a random interleaver $\Pi$ following a bit interleaved coded modulation (BICM) scheme [15]. We denote by c the interleaved codeword. $\mathbf{c}$ is mapped onto a Gray encoded $M$-QAM constellation where $M=2^{m}$ is the modulation order and $m$ denotes the number of bits per symbol. The mapper feeds a block of $\Psi \times T$ QAM symbols denoted by $S_{i}$ to the STBC MIMO encoder. Afterwards, STBC encoder output $\mathbf{X}$ is only transmitted via the selected best transmit antennas. The unselected transmit antennas are deactivated.

The MIMO channel is defined by the matrix $\mathbf{H}_{\left[N_{r} \times N_{t}\right]}$ where its entries $h_{i j}$ are assumed to be independent and identically distributed (i.i.d.) circularly symmetric complex Gaussian random variables with probability density function (pdf) $\sim C N(0,1) . C N\left(\mu, \sigma^{2}\right)$ denotes the complex Gaussian pdf with mean $\mu$ and variance $\sigma^{2} . h_{i j}$ is the channel coefficient between the the $j$-th transmit and the $i$-th receive antenna. A complex white Gaussian noise $\mathbf{N}_{\left[N_{r} \times T\right]}$ with i.i.d. entries and pdf $\sim C N\left(0, \sigma^{2}\right)$ is added to the received signals.

The channel input-output relation is given by:

$$
\mathbf{Y}=\mathbf{H X}+\mathbf{N}
$$

Receiver side: $N_{r} \times T$ noisy signals, denoted by $\mathbf{Y}_{\left[N_{r} \times T\right]}$, are received on the $N_{r}$ antennas. A soft ML STBC detector estimates the transmitted $M$-QAM symbols and feeds the $P\left(c_{i}=1\right) \quad\left(c_{i}\right.$ is the $i$-th bit of codeword $\left.\mathbf{c}\right)$, denoted by $\hat{c}_{i}$, to the deinterleaver. These probabilities are deinterleaved then provided to the FEC decoder. Resulting hard decoded information word $\hat{b}$ is compared to $\mathbf{b}$ to compute the BER.

In the sequel, we denote by $\Psi$ the set of best antennas. The cardinality $\psi$ of the set $\Psi$ is chosen depending on the tolerable complexity. The system transmitting over $\psi$ selected transmit antennas of $N_{t}$ is denoted by $\left(\psi / N_{t}\right) \times N_{r}$.

Antenna Selection Algorithm: The channel matrix is explicitly defined as:

$$
\mathbf{H}=\left[\begin{array}{cccc}
h_{11} & h_{12} & \cdots & h_{1 N_{t}} \\
h_{21} & h_{22} & \cdots & h_{2 N_{t}} \\
\vdots & \vdots & \ddots & \vdots \\
h_{N_{r} 1} & h_{N_{r} 2} & \cdots & h_{N_{r} N_{t}}
\end{array}\right]
$$

Depending on the channel conditions, many selection algorithms exist in the literature $[10-12,16]$. When a STBC is

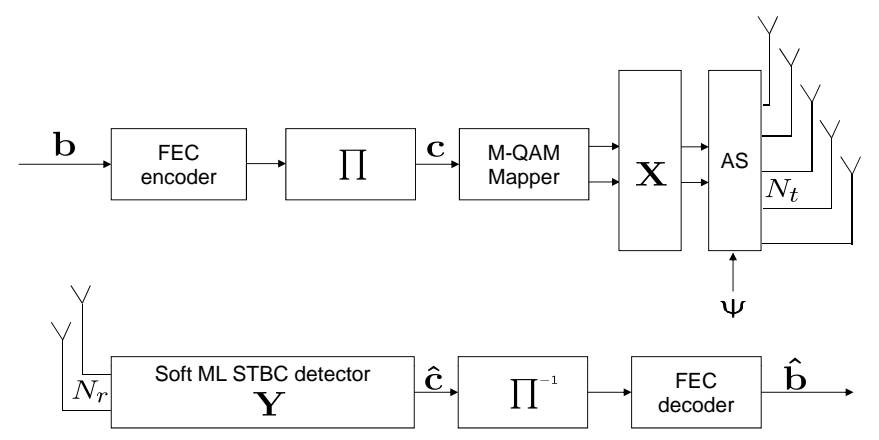

Fig. 1. Structure of the system at the transmitter and the receiver.

used, it is clear that the best AS algorithm is done by choosing the $\psi$ transmit antennas with highest norms $[12,14,16]$. Every $T$ periods, the receiver selects $\psi$ of $N_{t}$ columns as

$$
\max _{j \in\left\{1, \cdots, N_{t}\right\}-\Psi, j \leq \psi} \sum_{i=1}^{N_{r}}\left|h_{i j}\right|^{2}
$$

where $\Psi$ is the set of already selected antennas. When $j=$ $\psi$, the selected set $\Psi$ is fed to the transmitter. The selection algorithm requires a computation of the channel coefficients norms $\left|h_{i j}\right|^{2}$ and their summation. Its complexity is $\mathcal{O}\left(N_{t} N_{r}\right)$ which is small compared to the detection complexity equal to $\mathcal{O}\left(M^{N_{t} T}\right)$ [8] especially for high order modulations.

In the sequel, we consider the selection of dual antennas of the $N_{t}$ available ones $\left(\psi=2 \leq N_{t}\right)$ and we assess the transmit AS gains in the context of a turbo coded WiMAX system with respect to a classical $2 \times 2$ system as in [7, 8].

\section{STATE OF THE ART: $2 \times 2$ MIMO STBCS}

\section{A. WiMAX $2 \times 2$ MIMO profiles}

As an example of a $2 \times 2$ MIMO system, we consider the worldwide interoperability for microwave access (WiMAX) system which uses the MIMO codes specified in the IEEE 802.16e-2005 standard [17]. Two mandatory MIMO profiles are described for the downlink. The first represents the Alamouti code introduced for transmit diversity, referred to as matrix A (MA). It offers full-diversity (FD) but is only halfrate. The second profile is the full-rate (FR) spatial multiplexing introduced for spectral efficiency increase, referred to as matrix $\mathrm{B}(\mathrm{MB})$. In order to benefit from both diversity and multiplexing gains, another MIMO profile is included in the IEEE 802.16e-2005 specification, referred to as matrix C (MC) which is a variant of the Golden code [18].

\section{B. STBCs for $2 \times 2$ MIMO system}

Several FR-FD STBCs have been proposed for $2 \times 2$ systems [18-25]. Among them, the trace-orthonormal (TO) STBC has a flexible structure designed from both information-theoretic and detection error viewpoints [19]. By varying one of the code parameters, the performance of the TO STBC varies significantly. In [7], we have taken advantage of this feature in order to design a flexible TO-based STBC for $2 \times 2$ systems. The resulting structure has been shown to at least match and in some cases overcome existing STBCs for a wide range of SNRs and this without increasing the system complexity order. 


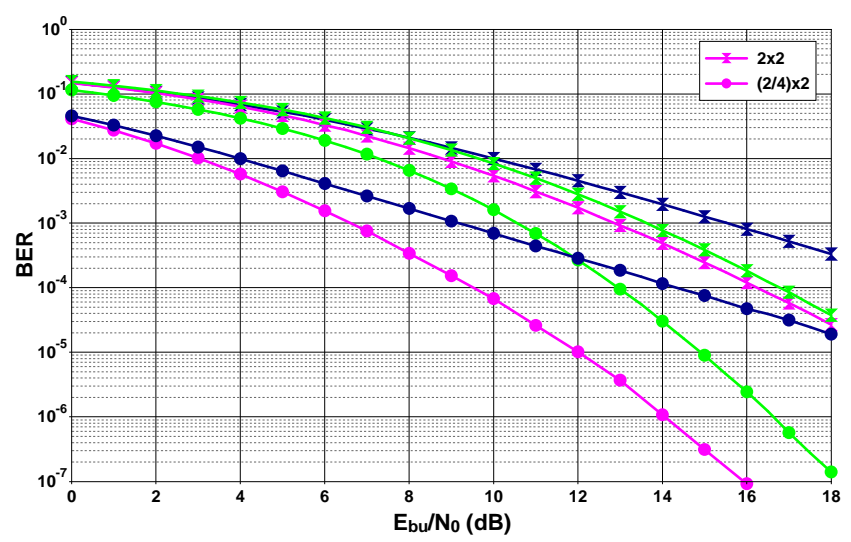

Fig. 2. Uncoded BER of $2 \times 2$ WiMAX profiles: MA with 16-QAM modulation, $\mathrm{MB}$ and $\mathrm{MC}$ with 4-QAM modulation; Dual transmit AS out of $N_{t}=2$ and 4; quasi-static flat Rayleigh fading MIMO channel.

\section{TRANSMIT AS FOR UNCODED MIDO SYSTEMS}

In this section, we assess the gain obtained by transmit AS technology for MIDO systems without any FEC codes. We consider the WiMAX profiles i.e., the Alamouti code (MA), the spatial multiplexing (MB) and the Golden code (MC). A fair comparison between FR MB and MC codes, and the half-rate MA code is done at the same spectral efficiency. Therefore, we use a $M$-QAM modulation for the FR profiles and $M^{2}$-QAM modulation for the MA.

Fig. 2 depicts uncoded BER curves as a function of $E_{b u} / N_{0}$ for MA with 16-QAM modulation in green curves, and for MB scheme in blue curves and MC in pink curves with 4-QAM modulation. $E_{b u}$ denotes the energy per bit for uncoded MIDO system and $N_{0}$ the noise power spectral density. Dual transmit antennas are selected out of $N_{t}=2$ and 4 .

Fig. 2 shows that the FD MA and MC profiles benefit from both diversity and SNR gains. The slope of their BER curves is steeper for higher number of available transmit antennas. Indeed, the FD profiles i.e., MA and MC benefit from all the available channel diversity as their BER curves slope is equal to $N_{t} N_{r}$ at high SNRs. Moreover, the FR-FD MC overcomes the MA as: 1) MA uses a 16-QAM modulation while MC uses a 4-QAM modulation and 2) MC benefits from MIMO coding gain. On the other hand, the slope of BER curves for the MB scheme remains the same with the increase of $N_{t}$. This result is expected as the MB scheme does not provide any transmit diversity. The MB scheme only benefits from SNR gains.

At a target BER equal to $10^{-5}$, the MC code overcomes the MA code with a gain of $3 \mathrm{~dB}$ and the $\mathrm{MB}$ code with $7 \mathrm{~dB}$ for $(2 / 4) \times 2$ system. The SNR gain between the FD profiles and the MB profile increases with the SNR as the slope of BER curves for the FD profiles is steeper than the MB one.

The transmit AS provides an important gain for MIDO systems that are not using powerful FEC codes. This gain is prominent at high SNRs. However, practical MIMO systems usually use such codes converging at low to moderate SNRs. In the sequel, we study the transmit AS gains for coded MIDO systems. We evaluate transmit AS gains in the context of a turbo coded WiMAX system.

\section{TRAnsmit AS For Coded MIMO Systems}

In this section, we recall the BMI criterion then we design adaptive STBC suitable for coded MIDO systems using transmit AS.

\section{A. BMI criterion and adaptive MIDO STBCs}

The asymptotic rank-determinant criteria optimize STBCs for high SNRs [2]. Most of practical communication systems are planned for low to moderate target SNRs due to the introduction of a powerful FEC code. Therefore, the STBC parameter optimization should target this SNR range. Based on the maximization of the BMI between transmitted and soft detected bits, we have proposed in $[7,8]$ a non-asymptotic design criterion aiming at choosing the appropriate design parameter value for STBCs at a specific target SNR. Definition At a SNR value, the BMI is computed as in [26], by:

$$
\begin{aligned}
& \operatorname{BMI}(\hat{c} ; c)=1-E\left[\log _{2}(1+\exp (-L))\right] \\
& \approx 1-\frac{1}{N} \sum_{n=1}^{N} \log _{2}\left(1+\exp \left(-u_{n} \cdot L_{n}\right)\right) \\
& \text { with }\left\{\begin{array}{l}
L_{n}=\ln \frac{1-\hat{c}_{n}}{\hat{c}_{n}} \\
u_{n}=(-1)^{c_{n}}
\end{array}\right.
\end{aligned}
$$

where $E$ is the mean function and $L$ denotes the log-likelihood ratio (LLR). $N$ is assumed to be large enough to accurately estimate the BMI. The BMI value is assessed by Monte Carlo simulations by passing a $N$-bit sequence into the mapper, the STBC, the MIMO channel and the soft ML detector.

\section{B. Adaptive Trace-Orthonormal STBC}

In this section, we design TO-based adaptive STBCs for each $\left(2 / N_{t}\right) \times N_{r}$ system according to the BMI criterion. The TO STBC is defined by the matrix $\mathrm{X}^{\mathrm{TO}}$. A group of 4 data symbols $\left(S_{1}, S_{2}, S_{3}, S_{4}\right)$ is transmitted as follows:

$$
\mathbf{X}^{\mathrm{TO}}=\frac{1}{\sqrt{2}}\left[\begin{array}{ll}
X_{11} & X_{12} \\
X_{21} & X_{22}
\end{array}\right]
$$

where

$$
\begin{aligned}
& X_{11}=\left(S_{1}+S_{2}\right) \cos \theta+\left(S_{2}^{*}-S_{1}^{*}\right) \sin \theta \\
& X_{12}=e^{\frac{j \pi}{4}}\left(\left(S_{3}+S_{4}\right) \sin \theta+\left(S_{4}^{*}-S_{3}^{*}\right) \cos \theta\right) \\
& X_{21}=e^{\frac{j \pi}{4}}\left(\left(S_{3}+S_{4}\right) \cos \theta+\left(S_{3}^{*}-S_{4}^{*}\right) \sin \theta\right) \\
& X_{22}=\left(S_{1}+S_{2}\right) \sin \theta+\left(S_{1}^{*}-S_{2}^{*}\right) \cos \theta
\end{aligned}
$$

$S^{*}$ designates the complex conjugate of $S$ and $\theta$ is the code design parameter to be optimized according to the selected criterion.

In [19], an exhaustive search is performed in order to maximize the minimum determinant. This search leads to a $\theta=\frac{1}{2} \arcsin \frac{1}{\sqrt{5}} \approx 13.28^{\circ}$ and a coding gain equal to the one of the MC code. We recall that the rank-determinant criteria [2] are suitable at high SNRs while practical communication systems operate in the range of low to moderate SNRs due to the introduction of powerful FEC codes.

In [7], we have shown that the BMI depends on $E_{b u} / N_{0}$ and the modulation order $M$. Therefore, we have computed for 


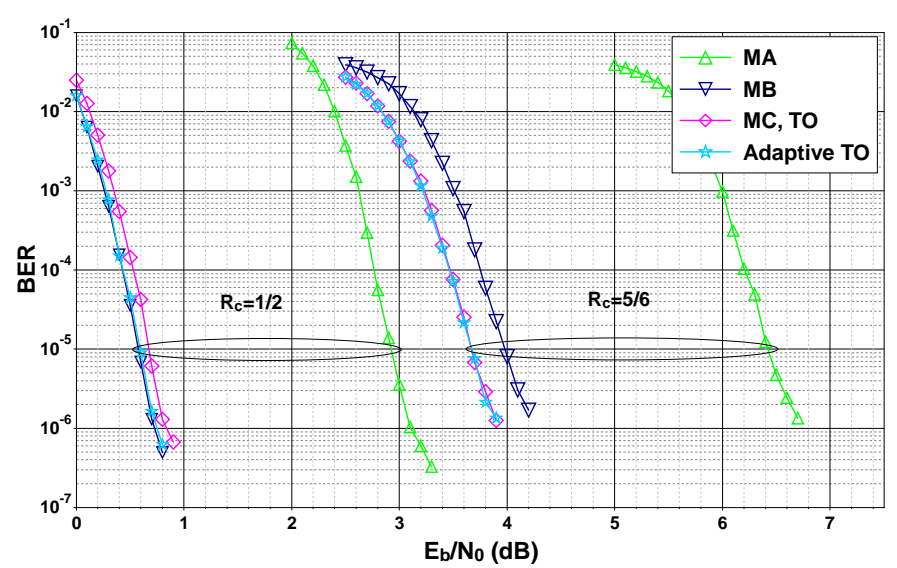

Fig. 3. BER of turbo coded $(2 / 3) \times 2$ system; 8 -state double binary turbo code $\left(R_{c}=1 / 2,5 / 6\right)$ with 8 turbo decoding iterations and information frame size $k=4,800$ bits; MIMO profiles: Alamouti code (MA) with 16-QAM modulation and spatial multiplexing (MB), Golden code (MC) and adaptive TO with 4-QAM modulation; quasi-static flat Rayleigh fading channel.

each $M$ and $E_{b u} / N_{0}$ with a step of $0.25 \mathrm{~dB}$, the appropriate $\theta$ maximizing the BMI of TO code. A polynomial interpolation has been performed to obtain an analytical approximation of obtained values. Following the same method [7], the design parameter $\theta$ of the TO STBCs is optimized for each $\left(2 / N_{t}\right) \times$ $N_{r}$ system as a function of $M$ and $E_{b u} / N_{0}$. We denote the obtained $\theta$ by $\theta_{\mathrm{opt}}^{M-\mathrm{QAM}}$.

For a 4-QAM modulation, the adaptive TO STBC design parameter is computed for $(2 / 3) \times 2$ by:

$$
\theta_{(2 / 3) \times 2}^{4-\mathrm{QAM}}=\left\{\begin{array}{c}
45^{\circ} ; \text { For } E_{b u} / N_{0} \leq-3.25 \mathrm{~dB} \\
0.52\left(\frac{E_{b u}}{N_{0}}\right)^{3}+2.51\left(\frac{E_{b u}}{N_{0}}\right)^{2}-5.74\left(\frac{E_{b u}}{N_{0}}\right) \\
+17 ; \text { For }-3.25<E_{b u} / N_{0}<0.75 \mathrm{~dB} \\
13.28^{\circ} ; \text { For } E_{b u} / N_{0} \geq 0.75 \mathrm{~dB}
\end{array}\right.
$$

And for a $(2 / 5) \times 2, \theta_{(2 / 5) \times 2}^{4-\mathrm{QAM}}$ is given by:

$$
\theta_{(2 / 5) \times 2}^{4-\mathrm{QAM}}=\left\{\begin{array}{c}
45^{\circ} ; \text { For } E_{b u} / N_{0} \leq-5.75 \mathrm{~dB} \\
0.23\left(\frac{E_{b u}}{N_{0}}\right)^{3}+4.85\left(\frac{E_{b u}}{N_{0}}\right)^{2}+18.26\left(\frac{E_{b u}}{N_{0}}\right) \\
+32.5 ; \text { For }-5.75<E_{b u} / N_{0}<-2.5 \mathrm{~dB} \\
13.28^{\circ} ; \text { For } E_{b u} / N_{0} \geq-2.5 \mathrm{~dB}
\end{array}\right.
$$

Based on the TO structure, an adaptive STBC can be designed for every $\left(2 / N_{t}\right) \times N_{r}$ system.

\section{Simulation results}

For the WiMAX system, the used FEC code is an 8-state double binary turbo code. In our simulations, such a code is used with rates $R_{c}=1 / 2$ and $5 / 6$ and an information frame size of 4,800 bits. The number of turbo-decoding iterations is equal to 8 . A random BICM interleaver is used. The number of receive antennas is $N_{r}=2$ and the number of selected antennas is $\psi=2$ out of $N_{t}=2,3,5$ transmit antennas. A 4-QAM modulation is used for the FR codes and a 16-QAM modulation for the Alamouti code (MA).

For the adaptive TO codes, the appropriate $\theta$ is computed as follows: 1) Passing from global system to uncoded MIMO

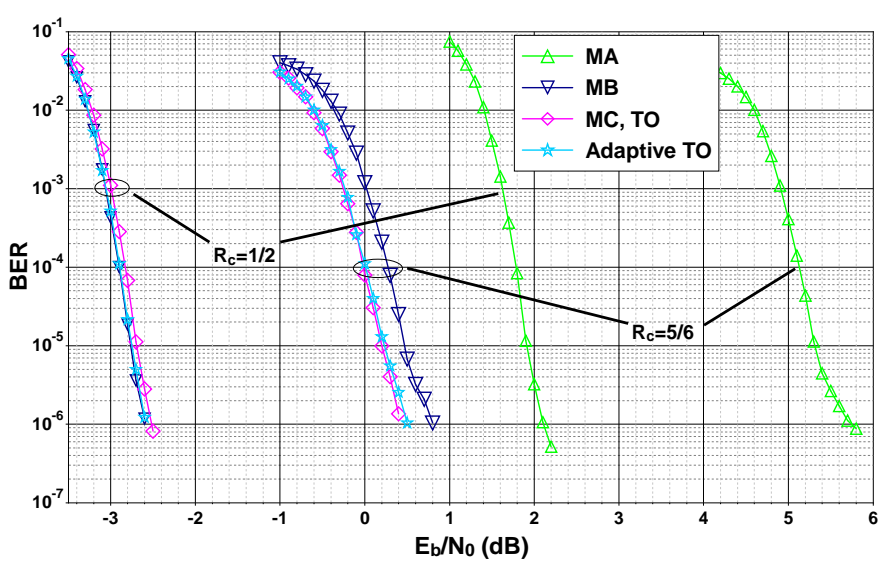

Fig. 4. BER of turbo coded $(2 / 5) \times 2$ system; 8 -state double binary turbo code $\left(R_{c}=1 / 2,5 / 6\right)$ with 8 turbo decoding iterations and information frame size $k=4,800$ bits; MIMO profiles: Alamouti code (MA) with 16-QAM modulation and spatial multiplexing (MB), Golden code (MC) and adaptive TO with 4-QAM modulation; quasi-static flat Rayleigh fading channel.

by: $E_{b u} / N_{0}=E_{b} / N_{0}+10 \log _{10}\left(R_{c}\right)$ where $E_{b}$ is the energy per information bit. 2) The obtained $E_{b u} / N_{0}$ is introduced in equation (7) for $(2 / 3) \times 2$ system and (8) for $(2 / 5) \times 2$ system. Therefore, the appropriate $\theta$ is obtained.

Fig. 3 and Fig. 4 plot the BER curves for $\psi=2$ selected transmit antennas out of $N_{t}=3$ and $N_{t}=5$ available ones, respectively. Both figures show that adaptive TO codes are more suitable for practical communication systems since they offer the best performance for all code rates. However, the use of classical non adaptive codes, i.e., MA, MB and MC codes introduces a performance loss up to $0.4 \mathrm{~dB}$ for some coding schemes.

In addition, we evaluate the gain of transmit $\mathrm{AS}$ with respect to the classical turbo coded $2 \times 2 \mathrm{WiMAX}$ system. We consider the best transmission for each system, i.e., its the adaptive TO code and a target BER equal to $10^{-5}$. The gain of $(2 / 3) \times 2$ system is more than $3 \mathrm{~dB}$ and the one of $(2 / 5) \times 2$ system is more than $6.35 \mathrm{~dB}$. This SNR gain increases with the total number of transmit antennas. Table I summarizes transmit AS gains for a $\left(2 / N_{t}\right) \times 2$ system with respect to classical turbo coded WiMAX system, for several number of antennas $N_{t}=$ $3,4,5$ and coding rates $R_{c}=1 / 2,5 / 6$.

On the other hand, the comparison between both figures shows that the AS technique does not provide any additional diversity gain as the same slope for BER curves, equal to the one for $2 \times 2$ system without AS (in [7]), is obtained at the same coding rate $R_{c}$. Indeed, the capacity-approaching FEC code achieves iterative convergence in the low SNR region where the effect of additional spatial diversity is not paramount. The AS gain is then obtained due to the obtained SNR gain.

Finally, the use of AS technique and adaptive TO codes is a promising approach for coded MIMO systems as it offers a substantial SNR gain with a low additional cost and complexity increase. 


\section{Discussion}

Authors in [27] consider transmit antenna and code selection (TACS) in the context of MIMO WiMAX systems. TACS consists of a selection, depending on the channel feedback, of best antennas and MIMO WiMAX code (MA, MB, MC) to be used for transmission. A similarity between [27] and our work exists, as we select also the best antennas and the parameter of adaptive TO codes based on the channel feedback. In contrast, an uncoded WiMAX system is considered in [27] and symbols are estimated based on a MMSE equalizer. Even if we consider their approach for coded WiMAX system and use a MLdetector as in our approach, our approach is more suitable for coded MIDO systems as: 1) it does not require a code switching and 2) can provide better performance for some cases. Indeed, depending on the parameter value, we have shown in this paper and in [7] that the adaptive TO codes can transmit based on MB code scheme at low SNRs, based on MC code scheme at high SNRs, and it overcomes both in some cases, providing advantage for the adaptive TO codes.

\begin{tabular}{|c|c|c|}
\hline MIMO system & $R_{c}=1 / 2$ & $R_{c}=5 / 6$ \\
\hline$(2 / 3) \times 2$ & 3 & 3.4 \\
\hline$(2 / 4) \times 2$ & 5.1 & 5.4 \\
\hline$(2 / 5) \times 2$ & 6.35 & 6.8 \\
\hline
\end{tabular}

TABLE I

GAINS IN DB OF CODED WIMAX MIDO SYSTEM USING TRANSMIT AS WITH RESPECT TO CLASSICAL $2 \times 2$ SYSTEM AT A TARGET BER $=10^{-5}$.

\section{CONCLUSIONS}

In this paper, we recalled the AS technology known as a technology providing MIMO gains with low cost and complexity. The performance of AS for MIMO system without powerful FEC codes is largely studied in the literature where FD STBCs can achieve the maximum diversity at high SNRs. However, coded MIMO systems operate at low to moderate SNRs. For each considered system, we have designed adaptive STBCs in a way overcoming conventional STBCs. In the context of a turbo coded WiMAX system, we have shown that AS technology provides a significant SNR gain of more than $3 \mathrm{~dB}$ by simply increasing the number of available transmit antennas $N_{t}$. This gain increases with the increase of $N_{t}$. Thanks to transmit AS technology, a substantial performance improvement can be obtained for coded MIMO systems with a low cost and complexity increase.

\section{REFERENCES}

[1] P. Wolniansky, G. Foschini, G. Golden, and R. Valenzuela, "V-BLAST: an architecture for realizing very high data rates over the rich-scattering wireless channel," in Proc. IEEE Int. Symp. on Signals, Syst. and Electronics (ISSSE), Sep. 1998.

[2] V. Tarokh, N. Seshadri, and A. Calderbank, "Space-time codes for high data rate wireless communications: Performance criterion and code construction," IEEE Trans. Inf. Theory, vol. 44, no. 2, pp. 744-765, Mar. 1998.

[3] S. Alamouti, "A simple transmit diversity technique for wireless communications," IEEE J. Sel. Areas in Commun., vol. 16, no. 8, pp. 14511458, Oct. 1998.
[4] L. Zheng and D. Tse, "Diversity and multiplexing: a fundamental tradeoff in multiple-antenna channels," IEEE Trans. Inf. Theory, vol. 49, no. 5, pp. 1073-1096, May 2003.

[5] A. El Falou, W. Hamouda, C. Langlais, C. Abdel Nour, and C. Douillard, "Finite-SNR diversity-multiplexing tradeoff for Rayleigh MIMO channels," IEEE Commun. Lett., vol. 17, no. 4, pp. 753-756, Apr. 2013.

[6] A. El Falou, C. Langlais, W. Hamouda, C. Abdel Nour, and C. Douillard, "Finite-SNR diversity-multiplexing tradeoff for spatially correlated Rayleigh MIMO channels," in Proc. IEEE Int. Conf. Commun. (ICC), to appear, Jun. 2014.

[7] A. El Falou, C. Langlais, C. Abdel Nour, and C. Douillard, "Adaptive trace-orthonormal STBC for MIMO system with capacity approaching FEC codes," in Proc. IEEE Vehicular Technology Conference (VTC), Sep. 2012.

[8] - "Low ML-detection complexity, adaptive $2 \times 2$ STBC, with powerful FEC codes," in Proc. Int. Symp. on Turbo Codes \& Iterative Information Processing, Aug. 2012.

[9] A. Sibille, C. Oestges, and A. Zanella, MIMO: From Theory to Implementation. Elsevier Inc., 2011.

[10] M. Win and J. Winters, "Analysis of hybrid selection/maximal-ratio combining in rayleigh fading," IEEE Trans. Commun., vol. 47, no. 12, pp. 1773-1776, Dec. 1999.

[11] R. Nabar, D. Gore, and A. Paulraj, "Optimal selection and use of transmit antennas in wireless systems," in Proc. Int. Conf. Telecommun.(ICT), 2000

[12] D. Gore and A. Paulraj, "Space-time block coding with optimal antenna selection," in Proc. IEEE Int. Conf. Acoust., Speech, and Signal Processing, May 2001.

[13] A. Molisch and M. Win, "MIMO systems with antenna selection," IEEE Microwave Mag., vol. 5, no. 1, pp. 46-56, 2004.

[14] F. Wang, X. Liu, and M. Bialkowski, "Space time block coded MIMO system with redundant antennas," in Int. Conf. on Information Assurance and Security (IAS), vol. 2, Aug. 2009, pp. 204-207.

[15] G. Caire, G. Taricco, and E. Biglieri, "Bit-interleaved coded modulation," IEEE Trans. Inf. Theory, vol. 44, no. 3, pp. 927 -946, May 1998.

[16] D. Gore and A. Paulraj, "MIMO antenna subset selection with spacetime coding," IEEE Trans. Signal Processing, vol. 50, no. 10, pp. 2580$2588,2002$.

[17] "IEEE 802.16-2005: IEEE standard for local and metropolitan area networks - Part 16: Air Interface for Fixed and Mobile Broadband Wireless Access Systems - Amendment 2: Physical Layer and Medium Access Control Layers for Combined Fixed and Mobile Operation in Licensed Bands," Feb. 2006.

[18] J.-C. Belfiore, G. Rekaya, and E. Viterbo, "The golden code: a $2 \times 2$ full-rate space-time code with nonvanishing determinants," IEEE Trans. Inf. Theory, vol. 51, no. 4, pp. 1432-1436, Apr. 2005.

[19] J.-K. Zhang, J. Liu, and K. Wong, "Trace-orthonormal full-diversity cyclotomic space-time codes," IEEE Trans. Signal Processing, vol. 55, no. 2, pp. 618-630, Feb. 2007.

[20] P. Dayal and M. Varanasi, "An optimal two transmit antenna spacetime code and its stacked extensions," IEEE Trans. Inf. Theory, vol. 51, no. 12, pp. 4348-4355, Dec. 2005.

[21] K. Srinath and B. Rajan, "A low-complexity, full-rate, full-diversity $2 \times 2$ STBC with golden code's coding gain," in Proc. IEEE Global Commun. Conf. (GLOBECOM), Dec. 2008.

[22] H. Yao and G. Wornell, "Achieving the full MIMO diversitymultiplexing frontier with rotation-based space-time codes," in Proc. Allerton Conf. Comm. Control and Computing, Oct. 2003.

[23] S. Sezginer and H. Sari, "Full-rate full-diversity $2 \times 2$ space-time codes of reduced decoder complexity," IEEE Commun. Lett., vol. 11, no. 12, pp. 973-975, Dec. 2007.

[24] J. Paredes, A. Gershman, and M. Gharavi-Alkhansari, "A new fullrate full-diversity space-time block code with nonvanishing determinants and simplified maximum-likelihood decoding," IEEE Trans. Signal Processing, vol. 56, no. 6, pp. 2461-2469, Jun. 2008.

[25] A. Hottinen, O. Tirkkonen, and R. Wichman, Multi-antenna Transceiver Techniques for $3 G$ and Beyond. John Wiley \& Sons, 2003.

[26] J. Hagenauer, "The EXIT chart-introduction to EXtrinsic Information Transfer in iterative processing," in Proc. European Signal Processing Conf., Sep. 2004, pp. 1541-1548.

[27] I. Gutierrez, F. Bader, and A. Mourad, "Joint transmit antenna and spacetime coding selection for WiMAX MIMO systems," in Proc. IEEE Int. Symp. on Personal, Indoor and Mobile Radio Commun. (PIMRC), 2009, pp. 3138-3143. 